\title{
Impact of Edible Coating and Different Packaging Treatments on Microbial Quality of Paneer
}

\section{Archana G. Lamdande ${ }^{1 *}$, Shyam R. Garud ${ }^{2}$ and Anil Kumar ${ }^{1}$}

${ }^{1}$ Deparment of Food Science and Technology, College of Agriculture, G.B.P.U.A.T., Pantnagar, India

${ }^{2}$ Deparment Post Harvest Process and Food Engineering, College of Technology, G.B.P.U.A.T., Pantnagar, India

\begin{abstract}
The effects of edible coating and different packaging treatments on microbial quality of paneer were studied Composite edible coated paneer was packed in packaging materials and stored under different storage conditions $5^{\circ} \mathrm{C}\left(\mathrm{T}_{1}\right), 30^{\circ} \mathrm{C}\left(\mathrm{T}_{2}\right)$ and ambient conditions $\left(\mathrm{T}_{3}\right)$. Packaging material had significant effect $(\mathrm{P} \leq 0.05)$, coating and temperature and their interactive effect were found significant $(P \leq 0.01)$ on total viable count of the product during storage. At $5 \pm 1{ }^{\circ} \mathrm{C}$, uncoated samples of paneer packed in laminates $\left(P_{4}\right)$ had total viable count $1.08 \times 10^{4} \mathrm{cfu} / \mathrm{g}$ on $28^{\text {th }}$ day of storage while coated paneer sample packed in LDPE $\left(P_{6}\right)$ and laminates $\left(P_{7}\right)$ had maximum shelf life i.e. 40 days with $1.6 \times 10^{3}$ and $2.75 \times 10^{3} \mathrm{cfu} / \mathrm{g}$ total viable count. Coating of paneer, packaging material and temperature and their interactive effect were found significant $(P \leq 0.01)$ on Yeast \& Mould count of the product during storage. Uncoated samples of paneer packed in laminates had $Y \& M$ counts $6.0 \times 10^{3} \mathrm{cfu} / \mathrm{g}$ on $28^{\text {th }}$ day of storage, whereas LDPE and laminates packed coated paneer had $Y \& M$ counts $3.4 \times 10^{3}$ and $2.15 \times 10^{3} \mathrm{cfu} / \mathrm{g}$ on $40^{\text {th }}$ day of storage $5 \pm 1^{\circ} \mathrm{C}$.
\end{abstract}

Keywords: Edible coating; Paneer; Quality; Packaging material; Shelf life

\section{Introduction}

Milk and milk products form an essential component of the human diet and no other single natural food meets the nutritional requirements better than milk. There are various dairy products like cheese, butter, ghee, paneer, khoa, curd; malai etc., among these paneer is an important indigenous dairy product. The shelf life of panner is only 1 day at room temperature and 6 days at $10^{\circ} \mathrm{C}[1]$. Many techniques have been studied in order to overcome these problems and extend the shelf life of fresh produce, for example, low temperature and high relative humidity, controlled and modified atmosphere packaging, etc. Edible film and coating enhances the quality of food products, protecting them from physical, chemical and microbiological deterioration [2]. Edible coating can act as moisture and gas barriers, control microbial growth, preserve the color, texture and moisture of the product, and can effectively extend the shelf life of the product.

The microbiological quality of paneer depends on the microbiological quality of milk, and the hygiene exercised during manufacture of paneer and its subsequent handling, packaging and storage. Microorganisms such as coliforms, yeasts and moulds that might be present in raw milk get destroyed completely, when milk is heated at $82^{\circ} \mathrm{C}$ for $5 \mathrm{~min}$. But these microbes may contaminate the product through a number of sources like air, water, equipment, knife, muslin cloth and persons handling the products [3]. These microbes can cause proteolytic and lipolytic changes, discoloration and other defects in the product [4]. According to Sachdeva [5], quality of paneer deteriorates due to the growth of organisms on the surface of paneer during storage. The total counts as well as yeast and mould counts increased during storage of paneer up to 10 days at $5^{\circ} \mathrm{C}$. Gupta [6], Thakral [7] and Parashar [8] reported about the increasing trend of the total viable count of paneer treated with $2 \%$ potassium sorbate.

The objective of present investigation is to evaluate the effect of edible coating and different packaging treatments on microbial quality of paneer and help to increase the shelf life of paneer.

\section{Materials and Methods}

Buffalo milk, whey protein concentrate, citric acid and water were used in the investigation.

\section{Paneer preparation}

Paneer was prepared as per the procedure outlined by Sachdeva [5]. Composite edible coating prepared by using whey protein concentrate and applied on the cubes of paneer. Edible coating acted as moisture and gas barrier, control microbial growth, preserve the color, texture and moisture of the product and effectively extend the shelf life of the product.

Then edible coated paneer cubes was packed into polypropylene, LDPE, and laminates and stored at temperatures $5 \pm 1^{\circ} \mathrm{C}, 30 \pm 1^{\circ} \mathrm{C}$ and ambient conditions. The stored paneer samples were tested periodically.

\section{Analytical procedures}

Total Viable count and Yeast and Mould Count were determined by using APHA [9] method.

\section{Statistical analysis}

Statistical analysis was performed using 3 Factorial completely randomized design (CRD). The experimental data were analyzed using the statistical methods of Snedecor and Cochran [10].

*Corresponding author: Archana G. Lamdande, Department of Food Science and Technology, College of Agriculture, G.B.P.U.A.T., Pantnagar, India, Tel: 09595284448; E-mail: archanalamdande@gmail.com

Received April 18, 2012; Accepted June 21, 2012; Published June 25, 2012

Citation: Lamdande AG, Garud SR, Anil Kumar (2012) Impact of Edible Coating and Different Packaging Treatments on Microbial Quality of Paneer. J Food Process Technol 3:159. doi:10.4172/2157-7110.1000159

Copyright: @ 2012 Lamdande AG, et al. This is an open-access article distributed under the terms of the Creative Commons Attribution License, which permits unrestricted use, distribution, and reproduction in any medium, provided the original author and source are credited. 
Citation: Lamdande AG, Garud SR, Anil Kumar (2012) Impact of Edible Coating and Different Packaging Treatments on Microbial Quality of Paneer. J Food Process Technol 3:159. doi:10.4172/2157-7110.1000159

Page 2 of 4

\section{Results and Discussion}

The effect of coating and packaging materials on microbiological characteristics of paneer during storage without vacuum were recorded below.

\section{Total Viable Count (TVC)}

Packaging material had significant effect $(\mathrm{P} \leq 0.05)$, whereas coating and temperature and their interactive effect were found significant $(\mathrm{P}$ $\leq 0.01$ ) on total viable count of the product during storage (Table 1 ).

The results showed that the initial total plate count of control sample of paneer $\left(\mathrm{P}_{1}\right)$ on zero days was $3.0 \times 10^{2} \mathrm{cfu} / \mathrm{g}$ and at the end of $2^{\text {nd }}$ day it was $4.6 \times 10^{3} \mathrm{cfu} / \mathrm{g}$. The uncoated samples of paneer packed in polypropylene $\left(\mathrm{P}_{2}\right)$, LDPE $\left(\mathrm{P}_{3}\right)$ and laminates $\left(\mathrm{P}_{4}\right)$ had microbial count $4.7 \times 10^{3}, 8.6 \times 10^{3}$ and $9.65 \times 10^{3} \mathrm{cfu} / \mathrm{g}$ on $4^{\text {th }}$ day of storage while coated samples of paneer packed in polypropylene $\left(\mathrm{P}_{5}\right)$, LDPE $\left(\mathrm{P}_{6}\right)$ and laminates $\left(\mathrm{P}_{7}\right)$ had microbial count $1.95 \times 10^{3}, 2.15 \times 10^{3}$ and $2.35 \times 10^{3}$ $\mathrm{cfu} / \mathrm{g}$ on $12^{\text {th }}$ day of storage at $30 \pm 1^{\circ} \mathrm{C}$ (Table 2$)$.

The initial total plate count of control fresh paneer $\left(\mathrm{P}_{1}\right)$ was $3.0 \times$ $10^{2} \mathrm{cfu} / \mathrm{g}$ which increased at the end of $6^{\text {th }}$ day up to $4.6 \times 10^{3} \mathrm{cfu} / \mathrm{g}$ Laminate packed uncoated paneer samples had microbial count 1.08 $\times 10^{4} \mathrm{cfu} / \mathrm{g}$ on $28^{\text {th }}$ day of storage. Whereas, coated samples of paneer packed in polypropylene had microbial count $2.15 \times 10^{3} \mathrm{cfu} / \mathrm{g}$ on $36^{\text {th }}$ day of storage and LDPE and laminates packed coated paneer had microbial count $1.6 \times 10^{3}$ and $2.75 \times 10^{3} \mathrm{cfu} / \mathrm{g}$ on $40^{\text {th }}$ day of storage at $5 \pm 1^{\circ} \mathrm{C}$ (Table 3 ).

\begin{tabular}{|c|c|c|c|c|c|c|c|}
\hline & a & b & c & $a^{*} b$ & $b^{*} \mathbf{c}$ & $a^{*} c$ & $a^{*} b^{*} c$ \\
\hline F value & 490.59 * & 5.49 * & $231.85^{\star *}$ & 9.32 ** & 33.30 ** & $193.42^{* *}$ & 37.82 ** \\
\hline SEM \pm & 19.56 & 23.96 & 23.96 & 33.88 & 33.88 & 41.50 & 58.69 \\
\hline C.D. at $5 \%$ & 56.23 & 68.86 & 68.86 & 97.38 & 97.38 & 119.27 & 168.68 \\
\hline C.D. at $1 \%$ & 75.24 & 92.15 & 92.15 & 130.32 & 130.32 & 159.61 & 225.72 \\
\hline
\end{tabular}

*Significant at $5 \%$ level of significance, ${ }^{* *}$ significant at $1 \%$ level of significance, ns - non significant, a- Coating/uncoating, b- packaging material, c- temperature Table 1: Effect of treatments on Total viable count of paneer during storage.

\begin{tabular}{|c|c|c|c|c|c|c|c|c|}
\hline \multirow{3}{*}{ Sample code } & \multicolumn{8}{|c|}{$\begin{array}{c}\text { Microbial Counts } \\
\text { (cfu/g) Total viable count }\end{array}$} \\
\hline & \multicolumn{8}{|c|}{ Days of storage } \\
\hline & 0 & 2 & 4 & 6 & 8 & 10 & 12 & 14 \\
\hline $\mathbf{P}_{1}$ & $3.0 \times 10^{2}$ & $4.6 \times 10^{3}$ & N.A & - & - & - & - & - \\
\hline $\mathbf{P}_{2}$ & $2.5 \times 10^{2}$ & $1.25 \times 10^{3}$ & $4.7 \times 10^{3}$ & N.A & - & - & - & - \\
\hline $\mathbf{P}_{3}$ & $2.0 \times 10^{2}$ & $1.35 \times 10^{3}$ & $8.6 \times 10^{3}$ & N.A & - & - & - & - \\
\hline $\mathbf{P}_{4}$ & $1.5 \times 10^{2}$ & $1.42 \times 10^{3}$ & $9.65 \times 10^{3}$ & N.A & - & - & - & - \\
\hline $\mathbf{P}_{5}$ & $2.0 \times 10^{2}$ & $4.5 \times 10^{2}$ & $7.0 \times 10^{2}$ & $8.0 \times 10^{2}$ & $1.25 \times 10^{3}$ & $1.55 \times 10^{3}$ & $1.95 \times 10^{3}$ & N.A \\
\hline$P_{6}$ & $1.0 \times 10^{2}$ & $3.5 \times 10^{2}$ & $7.5 \times 10^{2}$ & $9.5 \times 10^{2}$ & $1.45 \times 10^{3}$ & $1.66 \times 10^{3}$ & $2.15 \times 10^{3}$ & N.A \\
\hline $\mathbf{P}_{7}$ & $1.0 \times 10^{2}$ & $5.5 \times 10^{2}$ & $8.0 \times 10^{2}$ & $1.05 \times 10^{3}$ & $1.55 \times 10^{3}$ & $1.67 \times 10^{3}$ & $2.35 \times 10^{3}$ & N.A \\
\hline
\end{tabular}

$\mathrm{P}_{1}$ : Control (without coating \& without packaging)

$P_{3}$ : Uncoated + LDPE

$\mathrm{P}_{5}:$ : Coated + Polypropylene

$\mathrm{P}_{7}:$ Coated + Laminate
$\mathrm{P}_{2}$ : Uncoated + Polypropylene

$\mathrm{P}_{4}$ : Uncoated + Laminate

$P_{6}^{4}:$ Coated + LDPE

N.A.: Not Acceptable

Table 2: Effect of coating and packaging materials on Total viable count of paneer during storage at $30 \pm 1^{\circ} \mathrm{C}$.

\begin{tabular}{|c|c|c|c|c|c|c|c|c|c|c|c|c|}
\hline \multirow{3}{*}{$\begin{array}{l}\text { Sample } \\
\text { Code }\end{array}$} & \multicolumn{12}{|c|}{$\begin{array}{c}\text { Microbial Counts } \\
\text { (cfu/g) Total viable count }\end{array}$} \\
\hline & \multicolumn{12}{|c|}{ Days of storage } \\
\hline & 0 & 6 & 12 & 18 & 24 & 28 & 32 & 34 & 36 & 38 & 40 & 45 \\
\hline$P_{1}$ & $3.0 \times 10^{2}$ & $4.6 \times 10^{3}$ & N.A & - & - & - & - & - & - & - & - & - \\
\hline $\mathbf{P}_{2}$ & $2.5 \times 10^{2}$ & $9.5 \times 10^{2}$ & $5.5 \times 10^{3}$ & $6.3 \times 10^{3}$ & $7.0 \times 10^{3}$ & N.A & - & - & - & - & - & - \\
\hline$P_{3}$ & $2.0 \times 10^{2}$ & $8.5 \times 10^{2}$ & $1.9 \times 10^{3}$ & $3.0 \times 10^{3}$ & $5.5 \times 10^{3}$ & N.A. & - & - & - & - & - & - \\
\hline $\mathbf{P}_{4}$ & $1.5 \times 10^{2}$ & $4.2 \times 10^{2}$ & $2.75 \times 10^{3}$ & $6.55 \times 10^{3}$ & $8.5 \times 10^{3}$ & $1.08 \times 10^{4}$ & N.A. & - & - & - & - & - \\
\hline$P_{5}$ & $2.0 \times 10^{2}$ & $2.5 \times 10^{2}$ & $3.0 \times 10^{2}$ & $1.55 \times 10^{3}$ & $1.8 \times 10^{3}$ & $1.85 \times 10^{3}$ & $1.9 \times 10^{3}$ & $1.95 \times 10^{3}$ & $2.15 \times 10^{3}$ & N.A & - & - \\
\hline $\mathbf{P}_{6}$ & $1.0 \times 10^{2}$ & $3.5 \times 10^{2}$ & $4.2 \times 10^{2}$ & $6.8 \times 10^{2}$ & $1.1 \times 10^{3}$ & $1.25 \times 10^{3}$ & $1.3 \times 10^{3}$ & $1.45 \times 10^{3}$ & $1.5 \times 10^{3}$ & $1.55 \times 10^{3}$ & $1.6 \times 10^{3}$ & N.A. \\
\hline$P_{7}$ & $1.0 \times 10^{2}$ & $4.0 \times 10^{2}$ & $5.5 \times 10^{2}$ & $7.5 \times 10^{2}$ & $9.5 \times 10^{2}$ & $1.1 \times 10^{3}$ & $1.25 \times 10^{3}$ & $1.4 \times 10^{3}$ & $1.55 \times 10^{3}$ & $1.95 \times 10^{3}$ & $2.75 \times 10^{3}$ & N.A. \\
\hline \multicolumn{13}{|c|}{$\begin{array}{ll}\mathrm{P}_{1}: \text { Control (without coating \& without packaging) } & \mathrm{P}_{2}: \text { Uncoated + Polypropylene } \\
\mathrm{P}_{3}: \text { Uncoated + LDPE } & \mathrm{P}_{4} \text { : Uncoated + Laminate } \\
\text { N.A.: Not Acceptable } & \mathrm{P}_{5} \text { : Coated + Polypropylene } \\
\mathrm{P}_{6}: \text { Coated + LDPE } & \mathrm{P}_{7} \text { : Coated + Laminate }\end{array}$} \\
\hline
\end{tabular}

Table 3: Effect of coating and packaging materials on Total viable count of paneer during storage at $5 \pm 1^{\circ} \mathrm{C}$. 
Citation: Lamdande AG, Garud SR, Anil Kumar (2012) Impact of Edible Coating and Different Packaging Treatments on Microbial Quality of Paneer. J Food Process Technol 3:159. doi:10.4172/2157-7110.1000159

Page 3 of 4

The coated samples of paneer packed in polypropylene, LDPE and laminates had total plate count $5.95 \times 10^{3}, 5.15 \times 10^{3}$ and $6.55 \times 10^{3} \mathrm{cfu} / \mathrm{g}$ on $12^{\text {th }}$ day of storage under ambient conditions (Table 4 ).

\section{Yeast and Moulds counts ( $Y$ \& M counts)}

Coating of paneer, packaging material and temperature and their interaction had significant effect $(\mathrm{P} \leq 0.01)$ on $\mathrm{Y} \& \mathrm{M}$ counts of the product during storage at $30 \pm 1{ }^{\circ} \mathrm{C}$ (Tables 5 and 6 ). The uncoated samples packed in polypropylene, LDPE and laminates had Y \& M counts $1.25 \times 10^{3}, 1.85 \times 10^{3}$ and $2.05 \times 10^{3} \mathrm{cfu} / \mathrm{g}$ on $2^{\text {nd }}$ day. Coated paneer samples packed in polypropylene, LDPE and laminates had Y \& $\mathrm{M}$ counts $1.45 \times 10^{3}, 1.95 \times 10^{3}$ and $2.15 \times 10^{3} \mathrm{cfu} / \mathrm{g}$ on $12^{\text {th }}$ day of storage.

At $5 \pm 1{ }^{\circ} \mathrm{C}$, the uncoated samples packed in laminates had $\mathrm{Y} \& \mathrm{M}$ counts $6.0 \times 10^{3} \mathrm{cfu} / \mathrm{g}$ on $28^{\text {th }}$ day of storage (Table 7). Coated paneer samples packed in polypropylene had Y \& M counts $3.7 \times 10^{3} \mathrm{cfu} / \mathrm{g}$ on $36^{\text {th }}$ day, whereas LDPE and laminates had $3.4 \times 10^{3}$ and $2.15 \times 10^{3} \mathrm{cfu} / \mathrm{g}$ on $40^{\text {th }}$ day.

\begin{tabular}{|c|c|c|c|c|c|c|c|c|}
\hline \multirow{3}{*}{ Sample Code } & \multicolumn{8}{|c|}{$\begin{array}{c}\text { Microbial Counts } \\
\text { (cfu/g) Total viable count }\end{array}$} \\
\hline & \multicolumn{8}{|c|}{ Days of storage } \\
\hline & 0 & 2 & 4 & 6 & 8 & 10 & 12 & 14 \\
\hline $\mathbf{P}_{1}$ & $3.0 \times 10^{2}$ & $5.5 \times 10^{3}$ & N.A & - & - & - & - & - \\
\hline $\mathbf{P}_{2}$ & $2.5 \times 10^{2}$ & $4.0 \times 10^{3}$ & N.A & - & - & - & - & - \\
\hline $\mathbf{P}_{3}$ & $2.0 \times 10^{2}$ & $4.6 \times 10^{3}$ & N.A. & - & - & - & - & - \\
\hline $\mathbf{P}_{4}$ & $1.5 \times 10^{2}$ & $5.15 \times 10^{3}$ & N.A. & - & - & - & - & - \\
\hline$P_{5}$ & $2.0 \times 10^{2}$ & $7.5 \times 10^{2}$ & $1.15 \times 10^{3}$ & $2.15 \times 10^{3}$ & $3.72 \times 10^{3}$ & $5.15 \times 10^{3}$ & $5.95 \times 10^{3}$ & N.A \\
\hline $\mathbf{P}_{6}$ & $1.0 \times 10^{2}$ & $8.5 \times 10^{2}$ & $1.75 \times 10^{3}$ & $2.75 \times 10^{3}$ & $2.95 \times 10^{3}$ & $4.95 \times 10^{3}$ & $5.15 \times 10^{3}$ & N.A \\
\hline $\mathbf{P}_{7}$ & $1.0 \times 10^{2}$ & $7.5 \times 10^{2}$ & $2.15 \times 10^{3}$ & $2.85 \times 10^{3}$ & $4.75 \times 10^{3}$ & $5.45 \times 10^{3}$ & $6.55 \times 10^{3}$ & N.A \\
\hline
\end{tabular}

$\mathrm{P}_{1}$ : Control (without coating \& without packaging)

$\mathrm{P}_{3}$ : Uncoated + LDPE

$\mathrm{P}_{5}:$ Coated + Polypropylene

$P_{7}$ : Coated + Laminate

$\mathrm{P}_{2}:$ Uncoated + Polypropylene
$\mathrm{P}_{4}$ : Uncoated + Laminate
$\mathrm{P}_{6}$ : Cpspoated + LDPE
N.A.: Not Acceptable

$\mathrm{P}_{4}:$ Uncoated + Laminate

N.A.: Not Acceptable

Table 4: Effect of coating and packaging materials on Total viable count of paneer during storage at ambient conditions.

\begin{tabular}{|c|c|c|c|c|c|c|c|}
\hline & a & b & C & $a^{*} \mathbf{b}$ & $b^{*} c$ & $a^{*} c$ & $a^{*} b^{*} c$ \\
\hline F value & $\underset{* *}{380.01}$ & $\underset{* *}{160.68}$ & $\underset{* *}{342.02}$ & $\underset{* *}{150.21}$ & $\underset{* *}{86.27}$ & $\underset{* *}{186.86}$ & $\underset{* *}{80.41}$ \\
\hline SEM \pm & 14.77 & 18.09 & 18.09 & 25.59 & 25.59 & 31.35 & 44.33 \\
\hline C.D. at $5 \%$ & 42.38 & 51.91 & 51.91 & 73.41 & 73.41 & 89.91 & 127.16 \\
\hline C.D. at $1 \%$ & 56.83 & 69.61 & 69.61 & 98.44 & 98.44 & 120.56 & 170.50 \\
\hline
\end{tabular}

* Significant at $5 \%$ level of significance, ${ }^{* *}$ significant at $1 \%$ level of significance, ns - non significant, a- Coating of paneer, b- packaging material, c- temperature,

Table 5: Effect of treatments (without vacuum) on Yeast \& Mould count of paneer during storage.

\begin{tabular}{|c|c|c|c|c|c|c|c|c|}
\hline \multirow{3}{*}{ Sample Code } & \multicolumn{8}{|c|}{$\begin{array}{c}\text { Microbial Counts } \\
\text { (cfu/g) Yeast and Mould count }\end{array}$} \\
\hline & \multicolumn{8}{|c|}{ Days of storage } \\
\hline & 0 & 2 & 4 & 6 & 8 & 10 & 12 & 14 \\
\hline$P_{1}$ & $3.5 \times 10^{2}$ & $4.6 \times 10^{3}$ & N.A & - & - & - & - & - \\
\hline $\mathbf{P}_{2}$ & $2.5 \times 10^{2}$ & $1.25 \times 10^{3}$ & N.A & - & - & - & - & - \\
\hline $\mathbf{P}_{3}$ & $2.0 \times 10^{2}$ & $1.85 \times 10^{3}$ & N.A & - & - & - & - & - \\
\hline$P_{4}$ & $1.5 \times 10^{2}$ & $2.05 \times 10^{3}$ & N.A & - & - & - & - & - \\
\hline $\mathbf{P}_{5}$ & $2.0 \times 10^{2}$ & $3.5 \times 10^{2}$ & $7.5 \times 10^{2}$ & $9.5 \times 10^{2}$ & $1.15 \times 10^{3}$ & $1.25 \times 10^{3}$ & $1.45 \times 10^{3}$ & N.A. \\
\hline$P_{6}$ & $1.0 \times 10^{2}$ & $4.5 \times 10^{2}$ & $9.0 \times 10^{2}$ & $1.15 \times 10^{3}$ & $1.55 \times 10^{3}$ & $1.62 \times 10^{3}$ & $1.95 \times 10^{3}$ & N.A. \\
\hline$P_{7}$ & $2.0 \times 10^{2}$ & $3.5 \times 10^{2}$ & $1.12 \times 10^{3}$ & $1.45 \times 10^{3}$ & $1.75 \times 10^{3}$ & $1.85 \times 10^{3}$ & $2.15 \times 10^{3}$ & N.A. \\
\hline
\end{tabular}

$\mathrm{P}_{1}$ : Control (without coating \& without packaging)

$\mathrm{P}_{3}$ : Uncoated + LDPE

$\mathrm{P}_{2}:$ Uncoated + Polypropylene

$P_{5}$ : Coated + Polypropylene

$\mathrm{P}_{4}$ : Uncoated + Laminate

$P_{7}:$ Coated + Laminate

$P_{6}:$ Coated + LDPE

N.A.: Not Acceptable

Table 6: Effect of coating and packaging materials on Yeast and Mould count of paneer during storage at $30 \pm 1^{\circ} \mathrm{C}$. 
Citation: Lamdande AG, Garud SR, Anil Kumar (2012) Impact of Edible Coating and Different Packaging Treatments on Microbial Quality of Paneer. J Food Process Technol 3:159. doi:10.4172/2157-7110.1000159

Page 4 of 4

\begin{tabular}{|c|c|c|c|c|c|c|c|c|c|c|c|c|}
\hline \multirow{3}{*}{$\begin{array}{l}\text { Sample } \\
\text { Code }\end{array}$} & \multicolumn{12}{|c|}{$\begin{array}{c}\text { Microbial Counts } \\
\text { (cfu/g) Yeast and Mould count }\end{array}$} \\
\hline & \multicolumn{12}{|c|}{ Days of storage } \\
\hline & 0 & 6 & 12 & 18 & 24 & 28 & 32 & 34 & 36 & 38 & 40 & 42 \\
\hline$P_{1}$ & $3.5 \times 10^{2}$ & $3.6 \times 10^{3}$ & N.A & - & - & - & - & - & - & - & - & - \\
\hline $\mathbf{P}_{2}$ & $2.5 \times 10^{2}$ & $3.9 \times 10^{2}$ & $1.0 \times 10^{3}$ & $1.8 \times 10^{3}$ & $2.7 \times 10^{3}$ & N.A & - & - & - & - & - & - \\
\hline$P_{3}$ & $2.0 \times 10^{2}$ & $4.5 \times 10^{2}$ & $1.15 \times 10^{3}$ & $1.95 \times 10^{3}$ & $3.0 \times 10^{3}$ & N.A. & - & - & - & - & - & - \\
\hline $\mathbf{P}_{4}$ & $4.0 \times 10^{2}$ & $8.0 \times 10^{2}$ & $1.35 \times 10^{3}$ & $3.55 \times 10^{3}$ & $4.5 \times 10^{3}$ & $6.0 \times 10^{3}$ & N.A. & - & - & - & - & - \\
\hline$P_{5}$ & $2.0 \times 10^{2}$ & $3.5 \times 10^{2}$ & $3.5 \times 10^{2}$ & $4.5 \times 10^{2}$ & $2.65 \times 10^{3}$ & $2.85 \times 10^{3}$ & $2.95 \times 10^{3}$ & $3.2 \times 10^{3}$ & $3.7 \times 10^{3}$ & N.A & N.A & N.A \\
\hline$P_{6}$ & $1.0 \times 10^{2}$ & $2.5 \times 10^{2}$ & $8.0 \times 10^{2}$ & $1.4 \times 10^{3}$ & $1.8 \times 10^{3}$ & $1.85 \times 10^{3}$ & $1.9 \times 10^{3}$ & $2.2 \times 10^{3}$ & $2.4 \times 10^{3}$ & $3.25 \times 10^{3}$ & $3.4 \times 10^{3}$ & N.A. \\
\hline$P_{7}$ & $1.0 \times 10^{2}$ & $2.0 \times 10^{2}$ & $2.2 \times 10^{2}$ & $3.5 \times 10^{2}$ & $4.5 \times 10^{2}$ & $5.5 \times 10^{2}$ & $5.9 \times 10^{2}$ & $6.5 \times 10^{2}$ & $8.5 \times 10^{2}$ & $1.45 \times 10^{3}$ & $2.15 \times 10^{3}$ & N.A. \\
\hline \multicolumn{13}{|c|}{$\begin{array}{l}\mathrm{P}_{1}: \text { : Control (without coating \& without packaging) } \\
\mathrm{P}_{3}: \text { Uncoated + LDPE } \\
\mathrm{P}_{5}: \text { Coated + Polypropylene } \\
\mathrm{P}_{7}: \text { Coated + Laminate }\end{array}$} \\
\hline
\end{tabular}

Table 7: Effect of coating and packaging materials on Yeast and Mould count of paneer during storage at $5 \pm 1{ }^{\circ} \mathrm{C}$.

\begin{tabular}{|c|c|c|c|c|c|c|c|c|}
\hline \multirow{3}{*}{ Sample Code } & \multicolumn{8}{|c|}{$\begin{array}{c}\text { Microbial Counts } \\
\text { (cfu/g) Yeast and Mould count }\end{array}$} \\
\hline & \multicolumn{8}{|c|}{ Days of storage } \\
\hline & 0 & 2 & 4 & 6 & 8 & 10 & 12 & 14 \\
\hline $\mathbf{P}_{1}$ & $3.5 \times 10^{2}$ & $4.6 \times 10^{3}$ & N.A & - & - & - & - & - \\
\hline$P_{2}$ & $2.5 \times 10^{2}$ & $3.75 \times 10^{3}$ & N.A & - & - & - & - & - \\
\hline $\mathbf{P}_{3}$ & $2.0 \times 10^{2}$ & $6.3 \times 10^{3}$ & N.A & - & - & - & - & - \\
\hline $\mathbf{P}_{4}$ & $1.5 \times 10^{2}$ & $7.1 \times 10^{3}$ & N.A & - & - & - & - & - \\
\hline $\mathbf{P}_{5}$ & $2.0 \times 10^{2}$ & $4.5 \times 10^{2}$ & $1.35 \times 10^{3}$ & $1.75 \times 10^{3}$ & $2.22 \times 10^{3}$ & $4.75 \times 10^{3}$ & $6.15 \times 10^{3}$ & N.A. \\
\hline $\mathbf{P}_{6}$ & $1.0 \times 10^{2}$ & $3.5 \times 10^{2}$ & $1.15 \times 10^{3}$ & $1.65 \times 10^{3}$ & $2.45 \times 10^{3}$ & $3.75 \times 10^{3}$ & $4.35 \times 10^{3}$ & N.A. \\
\hline$P_{7}$ & $2.0 \times 10^{2}$ & $5.5 \times 10^{2}$ & $1.65 \times 10^{3}$ & $1.9 \times 10^{3}$ & $2.75 \times 10^{3}$ & $5.65 \times 10^{3}$ & $6.75 \times 10^{3}$ & N.A. \\
\hline
\end{tabular}

$P_{1}$ : Control (without coating \& without packaging)

$P_{3}$ : Uncoated + LDPE

$\mathrm{P}_{5}:$ Coated + Polypropylene

$\mathrm{P}_{7}$ : Coated + Laminate

$\mathrm{P}_{2}$ : Uncoated + Polypropylene

$\mathrm{P}_{4}$ : Uncoated + Laminate

$\mathrm{P}_{6}$ : Coated + LDPE

N.A.: Not Acceptable

Table 8: Effect of coating and packaging materials on Yeast and Mould count of paneer during storage at ambient conditions.

The Y \& M counts of paneer samples packed in polypropylene, LDPE and laminates was $6.15 \times 10^{3}, 4.35 \times 10^{3}$ and $6.75 \times 10^{3} \mathrm{cfu} / \mathrm{g}$ on $12^{\text {th }}$ day of storage at ambient conditions (Table 8 ).

\section{Acknowledgement}

Authors are grateful to All India Co-ordinated Research Project (AICRP) on Post Harvest Technology (PHT), for providing the financial assistance to carry out the research work. Technical support by the staff and facilities provided by the Department of Food Science \& Technology is duly acknowledged.

\section{References}

1. Bhattacharyya DC, Mathur ON, Srinivasan M, Samlik O (1971) Studies on the method of production and shelf life of paneer (Cooking Type of Acid Coagulated Cottage Cheese). J Food Sci Tech 8: 117-120.

2. Kester JJ, Fennema OR (1986) Edible Film and Coating: A Review. Ind J Dairy Sci 40: 42-49.

3. Aggarwal PK, Srinivasan RA (1980) Mould contamination from dairy and farm environment. Indian J Dairy Sci 33: 117-119
4. Thakral S, Gupta M, Ghodekar DR (1986) Microbiological quality of paneer Indian Dairyman 38: 388-390

5. Sachdeva S (1983) Production, packaging and preservation of paneer: Thesis Ph.D., Kurukhshtra University, Kurukhshtra.

6. Gupta M (1985) Studies on Food Poisoning Organism in Paneer. Thesis, M.Sc., Kurukshetra University, Kurukshetra.

7. Thakral S (1986) Studies on Yeast in Paneer. Thesis. M.Sc., Kurukshetra University, Kurukshetra.

8. Parashar MS (1987) Microbiological quality of preserved paneer. Thesis, M.Sc., Kurukshetra University, Kurukshetra.

9. American Public Health Association (1992) Standard methods for the examination of water and waste water. American Public Health Association, American water Work Association, and Water Pollution Control Federation, Washington, D.C.

10. Snedecor GW, Cochran WG (1968) Statistical methods. Oxford and IBH Publishing Company, New Delhi. 